\title{
Direct Arylation of Arene C-H Bonds by Cooperative Action of NHCarbene-Ruthenium(II) Catalyst and Carbonate via Proton Abstraction Mechanism.
}

Ismail Özdemir, ${ }^{[t]}$ Serpil Demir, ${ }^{[\dagger]}$ Bekir Çetinkaya, ${ }^{\left[{ }^{\dagger]}\right]}$ Christophe Gourlaouen, ${ }^{\text {[f] }}$ Feliu Maseras, $\left.{ }^{*}\right]$,' Christian Bruneau, ${ }^{[\S]}$ and Pierre $\mathrm{H}$. Dixneuf*[s]

\section{Supporting information Synthesis and DFT calculations 1. Synthesis}

\section{General procedure for the arylation of 2-phenylpyridine}

2-phenylpyridine (1) $(1.00 \mathrm{mmol})$ and aryl bromide $(2.20 \mathrm{mmol})$ were added to a solution of $\left[\mathrm{RuCl}_{2}(p \text {-cymene })\right]_{2}(0.025 \mathrm{mmol}), \mathrm{LH}^{+} \mathrm{Cl}^{-}(0.10 \mathrm{mmol})$ and $\mathrm{Cs}_{2} \mathrm{CO}_{3}(3.00 \mathrm{mmol})$ inNMethylpyrrolidone (NMP) $(2 \mathrm{~mL})$. The resulting mixture was stirred at $120{ }^{\circ} \mathrm{C}$ for $10-20 \mathrm{~h}$. $\mathrm{H}_{2} \mathrm{O}$ and EtOAc were added to the cold reaction mixture. The seperated organic phase was dried over $\mathrm{MgSO}_{4}$ and concentrated in vacuo. The remaining residue was purified by column chromatography on silica gel (pentane / diethylether) to yield 2,6-9. The spectral data were in accordance with those reported in the literature ${ }^{12 \mathrm{a}}$

\section{Preparation of 1,3-bis-(3,4,5-trimethoxybenzyl)benzimidazolium chloride (5a)}

To a solution of 1-(3,4,5-trimethoxybenzyl)benzimidazole( $2.98 \mathrm{~g}, 10.0 \mathrm{mmol})$ in DMF (5 $\mathrm{mL}$ ) was added slowly 3,4,5-(trimethoxybenzyl) chloride $(2.17 \mathrm{~g}, 10.0 \mathrm{mmol})$ and the resulting mixture was stirred at room temperature for $5 \mathrm{~h}$. Diethylether $(10 \mathrm{~mL})$ was added to obtain a white crystalline solid which was filtered off. The solid was washed with diethylether (3x $10 \mathrm{~mL})$ dried under vacuum and the crude product was recrystallized from ethanole / diethylether. $\mathrm{Mp}=248-249^{\circ} \mathrm{C}$, and the yield was $4.48 \mathrm{~g}, 87 \%, \mathrm{v}_{(\mathrm{CN})}=1603 \mathrm{~cm}^{-1}$.

Anal. Found: C, 63.00; H, 6.04; N: 5.49. Calc. for $\mathrm{C}_{27} \mathrm{H}_{31} \mathrm{~N}_{2} \mathrm{O}_{6} \mathrm{Cl}$ : C, 62.97; H, 6.07; N, 5.44.

${ }^{1} \mathrm{H}$ NMR $\left(\delta, 200.13 \mathrm{MHz}, \mathrm{DMSO}-\mathrm{d}_{6}\right): 3.75$ [s, $\left.12 \mathrm{H}, \mathrm{CH}_{2} \mathrm{C}_{6} \mathrm{H}_{2}\left(\mathrm{OCH}_{3}\right)_{3}-3,4,5\right] ; 3.61$ [s, 6H, $\left.\mathrm{CH}_{2} \mathrm{C}_{6} \mathrm{H}_{2}\left(\mathrm{OCH}_{3}\right)_{3}-3,4,5\right] ; \quad 5.66 \quad\left[\mathrm{~s}, \quad 4 \mathrm{H}, \quad \mathrm{CH}_{2} \mathrm{C}_{6} \mathrm{H}_{2}\left(\mathrm{OCH}_{3}\right)_{3}-3,4,5\right] ; \quad 7.00 \quad[\mathrm{~s}, \quad 4 \mathrm{H}$, $\left.\mathrm{CH}_{2} \mathrm{C}_{6} \mathrm{H}_{2}\left(\mathrm{OCH}_{3}\right)_{3}-3,4,5\right] ; 8.14\left[\mathrm{~m}, 2 \mathrm{H}, \mathrm{NC}_{6} \mathrm{H}_{4} \mathrm{~N}\right] ; 7.64\left[\mathrm{~m}, 2 \mathrm{H}, \mathrm{NC}_{6} \mathrm{H}_{4} \mathrm{~N}\right] ; 10.44[\mathrm{~s}, 1 \mathrm{H}$, $\mathrm{NCHN}] .{ }^{13} \mathrm{C}$ NMR $\left(\delta, 50 \mathrm{MHz}, \mathrm{DMSO}-\mathrm{d}_{6}\right): 56.8$ and $50.9\left[\mathrm{CH}_{2} \mathrm{C}_{6} \mathrm{H}_{2}\left(\mathrm{OCH}_{3}\right)_{3}-3,4,5\right] ; 60.6$ $\left[\mathrm{CH}_{2} \mathrm{C}_{6} \mathrm{H}_{2}\left(\mathrm{OCH}_{3}\right)_{3}-3,4,5\right] ; \quad 107.3,129.9 ; 131.7$ and $138.4 \quad\left[\mathrm{CH}_{2} \mathrm{C}_{6} \mathrm{H}_{2}\left(\mathrm{OCH}_{3}\right)_{3}-3,4,5\right]$; $114.7,127.4$ and $143.2\left[\mathrm{NC}_{6} \mathrm{H}_{4} \mathrm{~N}\right] ; 153.8[\mathrm{NCHN}]$.

Preparation of 1-(2,4,6-trimethylbenzyl)-3-(2-methoxyethyl)benzimidazolium chloride (5b)

Compound 5b was prepared in the same way as 5a from 1-(2,4,6trimethylbenzyl)benzimidazole $(2.50 \mathrm{~g}, 10.0 \mathrm{mmol})$ and 2-methoxyethyl chloride $(0.95 \mathrm{~g}$, $10.0 \mathrm{mmol})$ to give white crystals of $\mathbf{5 d}$ in $3.10 \mathrm{~g}, 90 \%$ yield, m.p. $=209-210{ }^{0} \mathrm{C}, \mathrm{v}_{(\mathrm{CN})}=$ $1616 \mathrm{~cm}^{-1}$. 
Anal. Found: C, 69.62; H, 7.28; N: 8.15. Calc. for $\mathrm{C}_{20} \mathrm{H}_{25} \mathrm{~N}_{2} \mathrm{OCl}$ : C, 69.65; H, 7.31; N, 8.12.

${ }^{1} \mathrm{H}$ NMR $\left(\delta, 200.13 \mathrm{MHz}, \mathrm{CDCl}_{3}\right): 2.22\left[\mathrm{~s}, 3 \mathrm{H}, \mathrm{CH}_{2} \mathrm{C}_{6} \mathrm{H}_{2}\left(\mathrm{CH}_{3}\right)_{3}-2,4,6\right] ; 2.25[\mathrm{~s}, 6 \mathrm{H}$, $\left.\mathrm{CH}_{2} \mathrm{C}_{6} \mathrm{H}_{2}\left(\mathrm{CH}_{3}\right)_{3}-2,4,6\right] ; 3.24$ [s, $\left.3 \mathrm{H}, \mathrm{CH}_{2} \mathrm{CH}_{2} \mathrm{OCH}_{3}\right] ; 3.84$ [ t, $2 \mathrm{H}, J=4.7 \mathrm{~Hz}, \mathrm{CH}_{2} \mathrm{CH}_{2} \mathrm{OCH}_{3}$ ]; $4.84\left[\mathrm{t}, 2 \mathrm{H}, J=4.7 \mathrm{~Hz} \mathrm{CH}_{2} \mathrm{CH}_{2} \mathrm{OCH}_{3}\right] ; 5.75\left[\mathrm{~s}, 2 \mathrm{H}, \mathrm{CH}_{2} \mathrm{C}_{6} \mathrm{H}_{2}\left(\mathrm{CH}_{3}\right)_{3}-2,4,6\right] ; 6.87$ [s, 2H, $\left.\mathrm{CH}_{2} \mathrm{C}_{6} \mathrm{H}_{2}\left(\mathrm{CH}_{3}\right)_{3}-2,4,6\right] ; 7.49\left[\mathrm{~m}, 4 \mathrm{H}, \mathrm{NC}_{6} \mathrm{H}_{4} \mathrm{~N}\right] ; 11.03$ [s, $\left.1 \mathrm{H}, \mathrm{NCHN}\right] .{ }^{13} \mathrm{C}$ NMR $(\delta, 50 \mathrm{MHz}$, $\left.\mathrm{CDCl}_{3}\right): 20.5$ and $21.4\left[\mathrm{CH}_{2} \mathrm{C}_{6} \mathrm{H}_{2}\left(\mathrm{CH}_{3}\right)_{3}-2,4,6\right] ; 47.5\left[\mathrm{CH}_{2} \mathrm{CH}_{2} \mathrm{OCH}_{3}\right] ; 48.1\left[\mathrm{CH}_{2} \mathrm{C}_{6} \mathrm{H}_{2}\left(\mathrm{CH}_{3}\right)_{3}-\right.$ $2,4,6] ; 59.4\left[\mathrm{CH}_{2} \mathrm{CH}_{2} \mathrm{OCH}_{3}\right] ; 70.6\left[\mathrm{CH}_{2} \mathrm{CH}_{2} \mathrm{OCH}_{3}\right] ; 125.4,130.6,138.3$ and 140.1 $\left[\mathrm{CH}_{2} \mathrm{C}_{6} \mathrm{H}_{2}\left(\mathrm{CH}_{3}\right)_{3}-2,4,6\right] ; 113.7,114.5,127.3,131.5,132.7$ and $143.9\left[\mathrm{NC}_{6} \mathrm{H}_{4} \mathrm{~N}\right] ; 144.1$ $[\mathrm{NCHN}]$.

The synthesis of $\mathbf{3 a - c}, \mathbf{4}$ and $\mathbf{5 c - d}$ azolium salts were reported in the respectively literature (1) (a) Ozdemir I.; Demir S.; Çetinkaya B.Tetrahedron 2005, 61, 9791-9798. (b) Ozdemir I.; Demir S.; Yaşar S.; Çetinkaya B. Appl. Organometal. Chem. 2005, 19, 55-58. (c) Ozdemir I.; Gök Y.; Gürbüz N.; Çetinkaya E.; Çetinkaya B. J. Heterocyclic Chem. 2005, 42, 303-306. (d ) Ozdemir I.; Gök Y.; Gürbüz N.; Yaşar S.; Çetinkaya E.; Çetinkaya B. Polish J. Chem. 2004, $78,2141-2147$.

\section{DFT Calculations}

Contents :

I. Computational details and references

II. Cartesian coordinates in Angstroms and potential energies in Hartrees

1. Mechanism with a

2. Mechanism with $b$

3. Mechanism with $\mathrm{c}$

4. Structures of $\mathrm{HCO}_{3}{ }^{-}, \mathrm{d}$, e and $\mathrm{f}$

III. Figure S1 


\section{Computational details and references}

Calculations have been performed using the GAUSSIAN03 package ${ }^{1}$ within B3LYP p $^{2,3}$ formalisms. The standard $6-31+\mathrm{G}(\mathrm{d}, \mathrm{p})^{4}$ basis set was used to describe the $\mathrm{H}, \mathrm{C}, \mathrm{N}$ and $\mathrm{O}$ atoms. The large core scalar relativistic pseudopotentials by Dolg et al. ${ }^{5}$ were used for Ru and the polarized LANL2DZ pseudopotential by Hay and $\mathrm{Wadt}^{6,7}$ for $\mathrm{Cl}$, coupled to a double-zeta quality basis set described by the (8s7p6d)/[6s5p3d] contraction for Ru and (3s4p1d)/[2s3p1d] contraction for $\mathrm{Cl}$ to describe the valence electrons.

Full geometry optimizations have been performed. The nature of the stationary points encountered has been characterized by a vibrational analysis performed within the harmonic approximation. Transition states were identified by the presence of one imaginary frequency. Each pathway was checked by connecting transition states to products and reactants following the displacement associated to the imaginary frequencies.

\footnotetext{
${ }^{1}$ GAUSSIAN 03, Revision C.02, M. J. Frisch, G. W. Trucks, H. B. Schlegel, G. E. Scuseria, M. A. Robb, J. R. Cheeseman, J. A. Montgomery, Jr., T. Vreven, K. N. Kudin, J. C. Burant, J. M. Millam, S. S. Iyengar, J. Tomasi, V. Barone, B. Mennucci, M. Cossi, G. Scalmani, N. Rega, G. A. Petersson, H. Nakatsuji, M. Hada, M. Ehara, K. Toyota, R. Fukuda, J. Hasegawa, M. Ishida, T. Nakajima, Y. Honda, O. Kitao, H. Nakai, M. Klene, X. Li, J. E. Knox, H. P. Hratchian, J. B. Cross, V. Bakken, C. Adamo, J. Jaramillo, R. Gomperts, R. E. Stratmann, O. Yazyev, A. J. Austin, R. Cammi, C. Pomelli, J. W. Ochterski, P. Y. Ayala, K. Morokuma, G. A. Voth, P. Salvador, J. J. Dannenberg, V. G. Zakrzewski, S. Dapprich, A. D. Daniels, M. C. Strain, O. Farkas, D. K. Malick, A. D. Rabuck, K. Raghavachari, J. B. Foresman, J. V. Ortiz, Q. Cui, A. G. Baboul, S. Clifford, J. Cioslowski, B. B. Stefanov, G. Liu, A. Liashenko, P. Piskorz, I. Komaromi, R. L. Martin, D. J. Fox, T. Keith, M. A. Al-Laham, C. Y. Peng, A. Nanayakkara, M. Challacombe, P. M. W. Gill, B. Johnson, W. Chen, M. W. Wong, C. Gonzalez, J. A. Pople, Gaussian, Inc., Wallingford CT (2004).

${ }^{2}$ C. Lee, W. Yang, R. G. Par, Phys. Rev. B 37, 785 (1988).

${ }^{3}$ A. D. Becke, J. Chem. Phys. 98, 5648 (1993).

${ }^{4}$ W.J. Hehre, R. Ditchfield and J.A. Pople, J. Chem. Phys. 56, 2257 (1972).

${ }^{5}$ W. Kuelche, M. Dolg, H. Stoll, H. Preuss, Mol. Phys. 74, 1245 (1991).

${ }^{6}$ P. J. Hay, W. R. Wadt, J. Chem. Phys. 82, 270, 284 and 299 (1985).

${ }^{7}$ C.E. Check, T.O. Faust, J.M. Bailey, B.J. Wright, T.M. Gilbert and L.S. Sunderlin, J. Phys. Chem. A, 105, 8111 (2001).
} 


\section{Cartesian coordinates in Angstroms and potential energies in Hartrees}

The listed cartesian coordinates correspond to optimizations performed at B3LYP level with SDD pseudopotential on ruthenium, LANL2DZdp on chlorine and $6-31+G(d, p)$ basis set on all other atoms.

\section{Mechanism with a}

\section{- 1t-a}

$\mathrm{E}=-909.17141908 \mathrm{Ha}$

$\begin{array}{lrrr}\mathrm{C} & 1.16736300 & -2.63754900 & -1.15136700 \\ \mathrm{C} & 2.28375700 & -3.42657600 & -0.83013800 \\ \mathrm{C} & 3.43791300 & -2.82304900 & -0.31952700 \\ \mathrm{C} & 3.48778100 & -1.44229000 & -0.09418900 \\ \mathrm{C} & 2.37595900 & -0.63426000 & -0.39336100 \\ \mathrm{C} & 1.24362700 & -1.26520800 & -0.93515300 \\ \mathrm{C} & 2.32853100 & 0.80480400 & -0.01220100 \\ \mathrm{C} & 3.48004000 & 1.56547100 & 0.21993700 \\ \mathrm{C} & 3.37387600 & 2.86306200 & 0.72547600 \\ \mathrm{C} & 2.10833100 & 3.36697400 & 1.02740000 \\ \mathrm{C} & 0.99237200 & 2.57738300 & 0.75432400 \\ \mathrm{~N} & 1.08865600 & 1.35265900 & 0.20337300 \\ \mathrm{H} & 0.26702000 & -3.07216800 & -1.57042500 \\ \mathrm{H} & 2.25722800 & -4.50138800 & -0.98701500 \\ \mathrm{H} & 4.30263500 & -3.43418600 & -0.07671900 \\ \mathrm{H} & 4.37854300 & -1.00838900 & 0.34968300 \\ \mathrm{H} & 4.45284100 & 1.13848400 & 0.00219000 \\ \mathrm{H} & 4.26653600 & 3.45882000 & 0.89086000 \\ \mathrm{H} & 1.97327900 & 4.35618300 & 1.45115700 \\ \mathrm{H} & -0.02607500 & 2.89993700 & 0.96054400 \\ \mathrm{Cl} & -1.89530300 & 2.36227100 & -0.77847400 \\ \mathrm{C} & -1.37495800 & -0.48663200 & 0.89168200 \\ \mathrm{~N} & -0.80270400 & -1.10546200 & 1.97863800 \\ \mathrm{C} & -1.76116800 & -1.59277400 & 2.85381900 \\ \mathrm{C} & -2.96656700 & -1.29670100 & 2.30709600 \\ \mathrm{~N} & -2.73038700 & -0.62053100 & 1.12613100 \\ \mathrm{C} & 0.62266400 & -1.26933400 & 2.24770800 \\ \mathrm{C} & -3.86623400 & -0.12501900 & 0.32301500 \\ \mathrm{H} & -1.49911200 & -2.10255700 & 3.76706700 \\ \mathrm{H} & -3.96792500 & -1.49458600 & 2.65514500 \\ \mathrm{H} & 0.73709500 & -1.56715500 & 3.29099900 \\ \mathrm{H} & 1.05838300 & -2.04131600 & 1.60944300 \\ \mathrm{H} & -4.16172100 & -0.87668800 & -0.40650800 \\ \mathrm{H} & -4.68326500 & 0.07983800 & 1.01954900 \\ \mathrm{H} & -3.58575200 & 0.80038000 & -0.17696600 \\ \mathrm{H} & 1.14097200 & -0.32517300 & 2.09242700 \\ \mathrm{Ru} & -0.57957400 & 0.41471100 & -0.68458300 \\ \mathrm{Cl} & -2.00713500 & -0.73421300 & -2.28095500 \\ \mathrm{H} & 0.44333800 & -0.69080200 & -1.58362000 \\ & & & \\ & & & \end{array}$

$\mathrm{E}=-909.126499503 \mathrm{Ha}$
$\mathrm{Ru}$
0.25961700
$-0.76973800$
0.59803700 


$\begin{array}{lrrr}\mathrm{C} 1 & 0.57504200 & 0.07666300 & 2.78074900 \\ \mathrm{C} 1 & 0.57780700 & -2.30779300 & -1.36906300 \\ \mathrm{C} & 3.26467800 & 0.90308600 & -1.87719800 \\ \mathrm{C} & 4.02058500 & 0.40810800 & -0.86591700 \\ \mathrm{H} & 3.54228600 & 1.42773600 & -2.77747100 \\ \mathrm{H} & 5.08721400 & 0.41821700 & -0.70793200 \\ \mathrm{~N} & 1.94230300 & 0.60887700 & -1.57732300 \\ \mathrm{~N} & 3.14929400 & -0.17919600 & 0.04114600 \\ \mathrm{C} & 1.86206100 & -0.06123500 & -0.39517900 \\ \mathrm{C} & 3.60053500 & -0.94398400 & 1.20817600 \\ \mathrm{H} & 3.00932600 & -0.67566000 & 2.08308800 \\ \mathrm{H} & 3.49451200 & -2.01357400 & 1.01141400 \\ \mathrm{H} & 4.65036200 & -0.70299900 & 1.38357700 \\ \mathrm{C} & 0.84011500 & 0.88665100 & -2.49781000 \\ \mathrm{H} & 0.04705500 & 0.16316700 & -2.32289200 \\ \mathrm{H} & 0.46888400 & 1.90553500 & -2.36072500 \\ \mathrm{H} & 1.20768100 & 0.76275800 & -3.51860500 \\ \mathrm{C} & -1.83229400 & 1.46502000 & -0.14681600 \\ \mathrm{C} & 0.14453900 & 2.51701900 & 0.48785400 \\ \mathrm{C} & -2.46559700 & 2.70862000 & -0.31053400 \\ \mathrm{C} & -0.41781700 & 3.78305700 & 0.35274400 \\ \mathrm{H} & 1.16584300 & 2.38793500 & 0.83037700 \\ \mathrm{C} & -1.74933800 & 3.87535100 & -0.06652100 \\ \mathrm{H} & -3.50479100 & 2.75648700 & -0.61519300 \\ \mathrm{H} & 0.17028400 & 4.66658500 & 0.57672100 \\ \mathrm{H} & -2.22465100 & 4.84432800 & -0.18838800 \\ \mathrm{C} & -2.46695400 & 0.15373600 & -0.27996200 \\ \mathrm{C} & -3.84596500 & -0.02339700 & -0.48323700 \\ \mathrm{C} & -1.63716300 & -0.97693900 & -0.07865600 \\ \mathrm{C} & -4.40637400 & -1.29912300 & -0.46071400 \\ \mathrm{H} & -4.49138000 & 0.83781100 & -0.62985800 \\ \mathrm{C} & -2.21133300 & -2.25663400 & -0.06331600 \\ \mathrm{H} & 0.87255400 & -2.12468600 & 1.13051700 \\ \mathrm{C} & -3.59201600 & -2.41197500 & -0.22922900 \\ \mathrm{H} & -5.47428000 & -1.42398600 & -0.61401400 \\ \mathrm{H} & -1.58272300 & -3.13041400 & 0.05645700 \\ \mathrm{H} & -4.02333300 & -3.40898900 & -0.20501500 \\ \mathrm{~N} & -0.53194400 & 1.39078400 & 0.22096600 \\ & & & \end{array}$

\section{- 3t-a}

\section{$E=-1173.6806294 \mathrm{Ha}$}

$\begin{array}{lr}\text { C } & 1.69640000 \\ \mathrm{C} & 2.90059500 \\ \mathrm{C} & 3.29175000 \\ \mathrm{C} & 2.47191700 \\ \mathrm{C} & 1.24937900 \\ \mathrm{C} & 0.88275100 \\ \mathrm{C} & 0.33530200 \\ \mathrm{C} & 0.25916100 \\ \mathrm{C} & -0.70931400 \\ \mathrm{C} & -1.60684800 \\ \mathrm{C} & -1.48026300 \\ \mathrm{~N} & -0.50088200 \\ \mathrm{H} & 1.37277300 \\ \mathrm{H} & 3.53193000 \\ \mathrm{H} & 4.23604400 \\ \mathrm{H} & 2.79821500 \\ \mathrm{H} & 0.92656100 \\ \mathrm{H} & -0.78641600\end{array}$

$\begin{array}{rr}-0.02625800 & -2.72179900 \\ 0.58796200 & -3.07295100 \\ 1.76702700 & -2.42812400 \\ 2.33792400 & -1.45165700 \\ 1.74252500 & -1.10329400 \\ 0.52913700 & -1.72836400 \\ 2.33264700 & -0.10658900 \\ 3.71296600 & 0.12026800 \\ 4.22452900 & 0.98082500 \\ 3.33263900 & 1.57321000 \\ 1.97238400 & 1.31798800 \\ 1.46499100 & 0.53459800 \\ -0.93853900 & -3.21305500 \\ 0.15108200 & -3.84241600 \\ 2.24140000 & -2.68255000 \\ 3.24012300 & -0.94180600 \\ 4.37793200 & -0.41724700 \\ 5.29454800 & 1.15157100\end{array}$




$\begin{array}{lr}\mathrm{H} & -2.41224900 \\ \mathrm{H} & -2.15146700 \\ \mathrm{C} 1 & -1.70708300 \\ \mathrm{C} & 1.27366500 \\ \mathrm{~N} & 1.68497900 \\ \mathrm{C} & 2.93311400 \\ \mathrm{C} & 3.34175700 \\ \mathrm{~N} & 2.33564200 \\ \mathrm{C} & 0.95527100 \\ \mathrm{C} & 2.47898700 \\ \mathrm{H} & 3.40334700 \\ \mathrm{H} & 4.23853900 \\ \mathrm{H} & 1.31359500 \\ \mathrm{H} & 1.12917100 \\ \mathrm{H} & 1.53471100 \\ \mathrm{H} & 3.28403700 \\ \mathrm{H} & 2.71775000 \\ \mathrm{H} & -0.10730600 \\ \mathrm{Cl} & -0.76152900 \\ \mathrm{H} & -0.17599800 \\ \mathrm{Ru} & -0.49350000 \\ \mathrm{C} & -3.49564000 \\ \mathrm{O} & -4.44325100 \\ \mathrm{O} & -2.30773600 \\ \mathrm{H} & -2.85941200 \\ \mathrm{O} & -3.70145200\end{array}$

3.67836500
1.23633800
-1.29175300
-0.97593000
-0.50378300
-0.99000700
-1.79001000
-1.77543100
0.41319400
-2.54220000
-0.73700700
-2.37705900
0.26791400
1.45232100
-2.53432900
-2.11781900
-3.58110800
0.17729000
-2.79440200
0.15698600
-0.53572500
-0.24347100
0.48574300
0.17371100
-2.04970700
-1.59857800

2.21315100

1.74455000

1.96542800

0.97737900

2.21229900

2. 56552000

1.55196100

0.59889500

3.08296100

$-0.63402800$

3.50304600

1.42789800

4.10592300

2.78762400

$-1.17272200$

$-1.24341100$

$-0.38441000$

3.04049200

$-0.98794100$

$-1.70786900$

0.02497900

$-1.26561800$

$-1.56926300$

$-0.95038300$

$-1.07141200$

$-1.27790800$

\section{- 4t-a}

\section{$\mathrm{E}=-1173.6584799 \mathrm{Ha}$}

\begin{tabular}{|c|c|c|c|}
\hline $\mathrm{C}$ & 0.30676700 & 1.10823700 & 2.71262500 \\
\hline $\mathrm{C}$ & -0.28192700 & 2.09654300 & 3.50419700 \\
\hline $\mathrm{C}$ & -1.07615400 & 3.08732200 & 2.91126400 \\
\hline C & -1.24038700 & 3.11108000 & 1.52441100 \\
\hline $\mathrm{C}$ & -0.62096100 & 2.13789700 & 0.72481500 \\
\hline $\mathrm{C}$ & 0.12572800 & 1.07730200 & 1.31624500 \\
\hline $\mathrm{C}$ & -0.65482600 & 2.16272900 & -0.74649600 \\
\hline $\mathrm{C}$ & -1.02981000 & 3.28025800 & -1.50673300 \\
\hline $\mathrm{C}$ & -0.89286400 & 3.26520300 & -2.89166100 \\
\hline $\mathrm{C}$ & -0.34930600 & 2.12635700 & -3.49490600 \\
\hline $\mathrm{C}$ & -0.01094000 & 1.03923400 & -2.69744000 \\
\hline $\mathrm{N}$ & -0.19501800 & 1.02565300 & -1.35898800 \\
\hline $\mathrm{H}$ & 0.92535100 & 0.33790300 & 3.16560400 \\
\hline $\mathrm{H}$ & -0.12865700 & 2.09670200 & 4.58123800 \\
\hline $\mathrm{H}$ & -1.55422400 & 3.84698500 & 3.52498800 \\
\hline $\mathrm{H}$ & -1.85590600 & 3.88832000 & 1.07769000 \\
\hline $\mathrm{H}$ & -1.39140000 & 4.16979600 & -1.00283500 \\
\hline $\mathrm{H}$ & -1.17350400 & 4.13251100 & -3.48295400 \\
\hline $\mathrm{H}$ & -0.18162800 & 2.07476000 & -4.56620500 \\
\hline $\mathrm{H}$ & 0.41749900 & 0.12907900 & -3.10596800 \\
\hline $\mathrm{Cl}$ & 1.01041100 & -2.10948400 & -1.99053100 \\
\hline $\mathrm{C}$ & -1.49695200 & -1.24179100 & 0.13104700 \\
\hline $\mathrm{N}$ & -2.44363900 & -1.49627700 & -0.84744100 \\
\hline $\mathrm{C}$ & -3.63433700 & -1.95717200 & -0.30985500 \\
\hline $\mathrm{C}$ & -3.46042100 & -2.00983400 & 1.03268000 \\
\hline $\mathrm{N}$ & -2.16944700 & -1.58012900 & 1.29040700 \\
\hline $\mathrm{C}$ & -2.27830400 & -1.31745900 & -2.28485500 \\
\hline $\mathrm{C}$ & -1.66989700 & -1.49643100 & 2.65994000 \\
\hline $\mathrm{H}$ & -4.47865900 & -2.22155300 & -0.92733600 \\
\hline $\mathrm{H}$ & -4.12423000 & -2.32847200 & 1.82126300 \\
\hline
\end{tabular}




$\begin{array}{lr}\mathrm{H} & -2.91714100 \\ \mathrm{H} & -2.56980100 \\ \mathrm{H} & -0.58362500 \\ \mathrm{H} & -1.99002300 \\ \mathrm{H} & -2.07377800 \\ \mathrm{H} & -1.23962100 \\ \mathrm{Cl} & 1.35530700 \\ \mathrm{H} & 1.34947100 \\ \mathrm{Ru} & 0.38389700 \\ \mathrm{C} & 3.56942800 \\ \mathrm{O} & 4.35346300 \\ \mathrm{O} & 2.33711200 \\ \mathrm{H} & 3.16656500 \\ \mathrm{O} & 3.94971400\end{array}$

-2.04225800
-0.30651500
-1.57005000
-0.55928900
-2.34190800
-1.51215000
-2.16085700
0.80869300
-0.54403100
0.19105500
0.76059500
0.60340900
-1.31000600
-0.90991600
-2.79801000
-2.58736800
2.64524000
3.12497800
3.22556400
-2.55006100
1.46878900
0.73164600
-0.12054800
0.16776000
0.90774600
-0.11322500
-0.95390800
-0.52650900

- 5t-a

$E=-1173.70248457 \mathrm{Ha}$
2.13817500
3. 41112400
4.05098200
3. 42103100
2.14790400
1.42847400
1.52398200
2.06880100
1.47213800
0.33582500
$-0.18576800$
0.35289900
1.65594600
3.91223200
5.03563100
3.93751900
2.97381400
1.89572600
$-0.14827900$
$-1.06980300$
$-2.46279700$
$-1.26773200$
$-2.15537700$
$-2.57241100$
$-1.95671100$
$-1.17622800$
$-2.64953600$
$-0.37607300$
$-3.27592100$
$-2.01540000$
$-3.67529700$
$-2.03065500$
$-0.33321100$
0.63252700
$-0.86927800$
$-2.65658900$
$-0.99232800$
1.65338000
$-0.37854800$
0.49214600
1.35068500
0.77119000 

$\mathrm{H}$
$\mathrm{O}$
$-3.27293000$
$-1.32428500$
0.43014100
$-4.07515300$
$-0.74037200$
0.24706800

\section{Mechanism with b}

\section{- 1t-b}

$E=-909.170125880 \mathrm{Ha}$

\begin{tabular}{|c|c|c|c|}
\hline $\mathrm{C}$ & -1.80915800 & -2.54238500 & 0.82050800 \\
\hline $\mathrm{C}$ & -3.14167800 & -2.94994700 & 0.81970500 \\
\hline $\mathrm{C}$ & -4.16009700 & -2.02022200 & 0.57276100 \\
\hline $\mathrm{C}$ & -3.84472100 & -0.68660300 & 0.31415500 \\
\hline $\mathrm{C}$ & -2.50955600 & -0.25052500 & 0.31335500 \\
\hline $\mathrm{C}$ & -1.48226300 & -1.20135400 & 0.55947500 \\
\hline C & -2.13093900 & 1.12447300 & -0.05531600 \\
\hline $\mathrm{C}$ & -3.00492100 & 2.21934200 & 0.00084700 \\
\hline $\mathrm{C}$ & -2.56547500 & 3.48023100 & -0.39220600 \\
\hline C & -1.24957100 & 3.62589200 & -0.84008600 \\
\hline $\mathrm{C}$ & -0.42242100 & 2.50883400 & -0.86928500 \\
\hline $\mathrm{N}$ & -0.83988100 & 1.28535700 & -0.47770100 \\
\hline $\mathrm{H}$ & -1.00457900 & -3.24926800 & 0.99121800 \\
\hline $\mathrm{H}$ & -3.38788600 & -3.99171200 & 1.00312100 \\
\hline $\mathrm{H}$ & -5.19820100 & -2.33869300 & 0.55968300 \\
\hline $\mathrm{H}$ & -4.64259300 & 0.00802800 & 0.07017000 \\
\hline $\mathrm{H}$ & -4.01707900 & 2.07936300 & 0.36264200 \\
\hline $\mathrm{H}$ & -3.23583600 & 4.33315600 & -0.34762400 \\
\hline $\mathrm{H}$ & -0.86340800 & 4.58607700 & -1.16497300 \\
\hline $\mathrm{H}$ & 0.59798000 & 2.55442200 & -1.23048300 \\
\hline $\mathrm{Ru}$ & 0.34485500 & -0.44226000 & -0.52910800 \\
\hline $\mathrm{H}$ & -0.46309300 & -0.88305300 & 1.00884600 \\
\hline $\mathrm{Cl}$ & 1.29897600 & -2.64919600 & -0.66474600 \\
\hline $\mathrm{C}$ & 2.76392500 & 1.15854800 & 2.54005200 \\
\hline C & 3.71820300 & 0.68904400 & 1.70270700 \\
\hline $\mathrm{H}$ & 2.83892800 & 1.66482600 & 3.48924800 \\
\hline $\mathrm{H}$ & 4.79381100 & 0.70417100 & 1.77963500 \\
\hline $\mathrm{Cl}$ & 1.35281600 & 0.31786000 & -2.53905100 \\
\hline $\mathrm{C}$ & 3.84155900 & -0.55357400 & -0.43906500 \\
\hline $\mathrm{H}$ & 4.11254100 & -1.55445900 & -0.09744200 \\
\hline $\mathrm{H}$ & 3.24568300 & -0.63138400 & -1.34266900 \\
\hline $\mathrm{H}$ & 4.73943600 & 0.03657700 & -0.64102200 \\
\hline $\mathrm{C}$ & 0.27478000 & 1.15340100 & 2.61598900 \\
\hline $\mathrm{H}$ & -0.41456100 & 1.67810000 & 1.95779500 \\
\hline $\mathrm{H}$ & -0.18850400 & 0.22409100 & 2.96130600 \\
\hline $\mathrm{H}$ & 0.47699600 & 1.78285300 & 3.48430100 \\
\hline $\mathrm{N}$ & 3.07452800 & 0.13300200 & 0.60862200 \\
\hline $\mathrm{N}$ & 1.54010800 & 0.88722400 & 1.94153100 \\
\hline C & 1.71117000 & 0.23651000 & 0.73546900 \\
\hline
\end{tabular}

\section{- 3t-b}

\section{$E=-1173.69616032 \mathrm{Ha}$}

$\begin{array}{lrrr}\mathrm{C} 1 & 0.76361900 & 0.14618900 & -2.54225300 \\ \mathrm{C} 1 & -0.32143900 & 0.50294000 & 2.25827700 \\ \mathrm{C} & 3.59112800 & -1.97490000 & 1.40599600 \\ \mathrm{C} & 4.25195800 & -1.11343600 & 0.59397500 \\ \mathrm{H} & 3.95281100 & -2.75601500 & 2.05660400 \\ \mathrm{H} & 5.30631500 & -0.99614100 & 0.39709800\end{array}$




$\begin{array}{lrrr}\mathrm{N} & 2.24059300 & -1.68231700 & 1.28766400 \\ \mathrm{~N} & 3.30228900 & -0.31256600 & -0.01654100 \\ \mathrm{C} & 2.02654400 & -0.63823400 & 0.40043600 \\ \mathrm{C} & 3.68710600 & 0.77109500 & -0.92456500 \\ \mathrm{H} & 2.89513100 & 0.92342300 & -1.65418000 \\ \mathrm{H} & 3.84289200 & 1.69555500 & -0.36552300 \\ \mathrm{H} & 4.60707600 & 0.46738300 & -1.43488800 \\ \mathrm{C} & 1.23584500 & -2.41011800 & 2.05560500 \\ \mathrm{H} & 0.30640000 & -1.84480000 & 2.04974000 \\ \mathrm{H} & 1.08681500 & -3.41410900 & 1.64078300 \\ \mathrm{H} & 1.57559100 & -2.49353800 & 3.09246600 \\ \mathrm{C} & -2.12297700 & -1.49841800 & -0.36247300 \\ \mathrm{C} & -0.14719700 & -2.6169000 & -0.88827000 \\ \mathrm{C} & -2.85667100 & -2.65492500 & -0.67053900 \\ \mathrm{C} & -0.82307400 & -3.78593000 & -1.20500900 \\ \mathrm{H} & 0.92578200 & -2.54569800 & -1.00274900 \\ \mathrm{C} & -2.21476200 & -3.81957200 & -1.07830800 \\ \mathrm{H} & -3.93921500 & -2.61010500 & -0.62420300 \\ \mathrm{H} & -0.26088400 & -4.64422600 & -1.56001000 \\ \mathrm{H} & -2.78358200 & -4.71170600 & -1.32464800 \\ \mathrm{C} & -2.82023000 & -0.23923200 & -0.02261900 \\ \mathrm{C} & -4.01264100 & -0.25316000 & 0.72322500 \\ \mathrm{C} & -2.30867100 & 0.99876000 & -0.44703500 \\ \mathrm{C} & -4.67276800 & 0.93601000 & 1.02990800 \\ \mathrm{H} & -4.39920400 & -1.19386900 & 1.10542200 \\ \mathrm{C} & -2.95953500 & 2.19156900 & -0.12651700 \\ \mathrm{H} & -1.43869700 & 1.06232400 & -1.11262400 \\ \mathrm{C} & -4.14769300 & 2.16227000 & 0.60572800 \\ \mathrm{H} & -5.58291200 & 0.90707300 & 1.62442200 \\ \mathrm{H} & -2.49622900 & 3.12193000 & -0.43498800 \\ \mathrm{H} & -4.65239000 & 3.08993800 & 0.86382000 \\ \mathrm{~N} & -0.75378600 & -1.49142200 & -0.42828500 \\ \mathrm{Ru} & 0.34002100 & 0.23310400 & -0.09383800 \\ \mathrm{O} & 1.38033800 & 2.07371900 & 0.10274900 \\ \mathrm{C} & 0.90346900 & 3.20046400 & -0.23322900 \\ \mathrm{O} & -0.20932000 & 3.50879000 & -0.68713500 \\ \mathrm{O} & 1.83316400 & 4.23004600 & -0.04801400 \\ \mathrm{H} & 1.36017800 & 5.02244700 & -0.33861400\end{array}$

\section{- 4t-b}

\section{$E=-1173.65197725 \mathrm{Ha}$}

$\begin{array}{lr}\mathrm{Cl} & -0.62391000 \\ \mathrm{Cl} & 0.00972800 \\ \mathrm{C} & -3.90055400 \\ \mathrm{C} & -4.41360100 \\ \mathrm{H} & -4.38503500 \\ \mathrm{H} & -5.43540100 \\ \mathrm{~N} & -2.52086000 \\ \mathrm{~N} & -3.33841300 \\ \mathrm{C} & -2.13344400 \\ \mathrm{C} & -3.52020600 \\ \mathrm{H} & -2.69978300 \\ \mathrm{H} & -3.53378300 \\ \mathrm{H} & -4.46854200 \\ \mathrm{C} & -1.64185100 \\ \mathrm{H} & -0.62479800 \\ \mathrm{H} & -1.70574000 \\ \mathrm{H} & -1.93904500 \\ & \end{array}$

-0.24189000
-0.43751900
1.57158400
0.61088100
2.31353800
0.35003300
1.45744600
-0.06437800
0.43287900
-1.21088200
-1.23680700
-2.14034200
-1.08247600
2.32820500
1.95255000
3.35740400
2.29969000
-2.45238300
2.50090800
1.30917100
0.50018500
1.92520700
0.27095800
1.23620300
-0.05436700
0.39324700
-0.94391700
-1.65998500
-0.36956600
-1.47520800
2.00945800
1.94022500
1.63603400
3.06168000 
C
C
C
C
H
C
H
H
H
C
C
C
C
H
C
H
C
H
H
H
N
Ru
O
C
O
O
H
1.98496100

$-0.09757900$

2. 61587200

0.47552700

$-1.17153600$

1.87007600

3.69680300

$-0.16591200$

2. 35929100

2.70235400

4.03859300

1.96948800

4.69314400

4.55745700

2.66729900

1.13632200

3.98753900

5. 72185600

2.15095200

4.46642400

0.61846000

$-0.22322300$

$-0.97834500$

$-0.28377200$

0.85370800

$-0.75067800$

$-0.09145900$
1. 62435700

2.60909100

2.76448300

3.76196300

2.48797400

3.84834800

2.77396800

4.55988800

4.72501400

0.44168500

0.55794000

$-0.77348300$

$-0.51592500$

1. 51017100

$-1.81918400$

$-1.69916200$

$-1.71074200$

$-0.41426700$

$-2.75858700$

$-2.55334000$

1.56014300

$-0.26284900$

$-2.33531400$

$-3.08568900$

$-2.80399100$

$-4.34180900$

$-4.76655400$
$-0.47609000$

$-0.86296400$

$-1.00272800$

$-1.37923400$

$-0.83480400$

$-1.44866400$

$-1.08558000$

$-1.74046100$

$-1.86470900$

0.00718600

0.44242900

0.14413200

1.03628700

0.36090000

0.79839400

$-0.75101600$

1.22923700

1. 37419600

0.97199800

1.72497700

$-0.38909300$

0.02699300

0.05102200

$-0.65377100$

$-1.17721700$

$-0.89808500$

$-1.46657700$

\section{- 5t-b}

\section{$E=-1173.66734296 \mathrm{Ha}$}

\begin{tabular}{|c|c|c|c|}
\hline $\mathrm{Cl}$ & 0.12325100 & 0.57511300 & -2.41665700 \\
\hline $\mathrm{Cl}$ & 0.05629100 & -0.05935800 & 2.57271200 \\
\hline $\mathrm{C}$ & 4.09486100 & -1.75187700 & 0.75482100 \\
\hline $\mathrm{C}$ & 4.48166800 & -0.74133400 & -0.06373600 \\
\hline $\mathrm{H}$ & 4.66900300 & -2.51777700 & 1.25395900 \\
\hline $\mathrm{H}$ & 5.45940100 & -0.45028000 & -0.41703200 \\
\hline $\mathrm{N}$ & 2.71702200 & -1.65553200 & 0.88106700 \\
\hline $\mathrm{N}$ & 3.32719200 & -0.06156500 & -0.42121100 \\
\hline $\mathrm{C}$ & 2.19320100 & -0.59740100 & 0.15957900 \\
\hline $\mathrm{C}$ & 3.35817800 & 1.13480900 & -1.25206900 \\
\hline $\mathrm{H}$ & 2.42627600 & 1.20030200 & -1.81196000 \\
\hline $\mathrm{H}$ & 3.49022200 & 2.02955300 & -0.63431500 \\
\hline $\mathrm{H}$ & 4.19393700 & 1.05549500 & -1.95462800 \\
\hline $\mathrm{C}$ & 1.96147100 & -2.59702900 & 1.70108300 \\
\hline $\mathrm{H}$ & 0.95731000 & -2.20527600 & 1.84923600 \\
\hline $\mathrm{H}$ & 1.93502500 & -3.58393600 & 1.22325300 \\
\hline $\mathrm{H}$ & 2.43771800 & -2.68311200 & 2.68253400 \\
\hline $\mathrm{C}$ & -2.17963200 & -1.60233800 & -0.29501800 \\
\hline $\mathrm{C}$ & -0.18223300 & -2.71346000 & -0.80761200 \\
\hline $\mathrm{C}$ & -2.90027700 & -2.75596800 & -0.64886300 \\
\hline $\mathrm{C}$ & -0.85089800 & -3.87007700 & -1.18839800 \\
\hline $\mathrm{H}$ & 0.89482400 & -2.64105500 & -0.88216400 \\
\hline C & -2.24642600 & -3.90084600 & -1.08878800 \\
\hline $\mathrm{H}$ & -3.98320000 & -2.73460800 & -0.59606700 \\
\hline $\mathrm{H}$ & -0.28367500 & -4.71935700 & -1.55703500 \\
\hline $\mathrm{H}$ & -2.80977200 & -4.78780800 & -1.36712700 \\
\hline $\mathrm{C}$ & -2.77162600 & -0.32217400 & 0.08314200 \\
\hline C & -4.15834100 & -0.13511000 & 0.2441920 \\
\hline
\end{tabular}


$\mathrm{C}$
$\mathrm{C}$
$\mathrm{H}$
$\mathrm{C}$
$\mathrm{H}$
$\mathrm{C}$
$\mathrm{H}$
$\mathrm{H}$
$\mathrm{H}$
$\mathrm{N}$
$\mathrm{R}$
$\mathrm{O}$
$\mathrm{C}$
$\mathrm{O}$
$-1.83090800$

$-4.65907400$

$-4.85408600$

$-2.38621100$

0.07080000

$-3.75897100$

$-5.72894800$

$-1.72532800$

$-4.13771300$

$-0.80144500$

0.13639900

0.89552000

0.70923500

0.22523600

1.02790400

0.80166900
0.73358700

1.11564200

$-0.96114800$

1.97917800

2. 38446500

2.17560100

1. 26444000

2. 81851200

3.15480500

$-1.60740800$

0.14416500

2.23566800

3. 23758100

3.32006900

4.45545900

5.11496900
0.26903000

0.59570900

0.10634600

0.64173500

$-1.80098800$

0.79737500

0.72342100

0.84933100

1.08981400

$-0.33636300$

0.11086800

0.47683200

$-0.19207900$

$-1.41896100$

0.31075900

$-0.36180400$

\section{Mechanism with c}

\section{- 1t-c}

\section{$E=-909.16102109 \mathrm{Ha}$}

C
C
C
C
C
C
C
C
C
C
C
N
H
H
H
H
H
H
H
H
Cl
C
N
C
C
N
C
C
H
H
H
H
H
H
H
Cl

0.40724900

0.04418700

$-1.16541500$

$-2.00347400$

$-1.65549900$

- 0.41809300

$-2.46174700$

$-3.80844500$

$-4.48123500$

$-3.79027600$

$-2.45944100$

$-1.79948000$

1. 33267800

0.69552000

- 1.45195800

$-2.92200400$

$-4.32436900$

$-5.52672800$

$-4.27294000$

$-1.87759500$

0.71938800

2.10347500

2.45994000

3.83464300

4.34832400

3. 27183000

1. 51529800

3. 39671100

4.31632700

5.36583900

0.85350900

2.07422700

2.40680700

4.05268600

3. 80121200

0.92470800

$-0.71295000$
2.53125300

3.84825300

4.11864900

3.06953100

1.73724100

1.46090100

0.59414600

0.67318900

$-0.46782200$

$-1.68145800$

$-1.71491400$

$-0.60072200$

2. 31975800

4.66399200

5.14321300

3.29204200

1. 62446400

$-0.41405400$

$-2.60277500$

- 2.62725600

- 2.54491700

0.04929800

0.66728000

0.86207400

0.34904000

$-0.13720400$

1.03763600

$-0.87574500$

1.33943200

0.28140600

0.19179700

1. 27754900

- 0.98284800

$-0.33195700$

$-1.87405600$

1.90616500

- 2.02445800
$-1.27875600$

$-1.00504700$

$-0.35121300$

0.02685300

$-0.24559400$

- 0.88462100

0.19780100

0.57381600

0.99940100

1. 03042100

0.63335000

0.24269400

$-1.80545300$

$-1.30578400$

$-0.13358100$

0.56131500

0.51156400

1. 28692400

1. 33794800

0.62448600

1. 04354900

0.32554700

1.47995600

1.53716500

0.38913900

$-0.34388600$

2. 53156400

$-1.59980600$

2. 37600300

0.03729700

2. 72724400

3.43805600

$-2.04795700$

$-2.28494600$

$-1.41152000$

2. 22799000

$-2.07718800$ 


$\begin{array}{lrrr}\mathrm{Ru} & 0.15383300 & -0.66146500 & -0.31672000 \\ \mathrm{H} & -0.28961500 & 0.52382800 & -1.56348600\end{array}$

\section{- 2t-c}

\section{$\mathrm{E}=-909.1565275 \mathrm{Ha}$}

\begin{tabular}{|c|c|c|c|}
\hline $\mathrm{C}$ & -0.26082000 & 2.51954100 & -0.96469200 \\
\hline $\mathrm{C}$ & -1.08042400 & 3.64299800 & -1.09940900 \\
\hline $\mathrm{C}$ & -2.43594200 & 3.57810600 & -0.75745900 \\
\hline $\mathrm{C}$ & -2.96381600 & 2.37199000 & -0.30873300 \\
\hline $\mathrm{C}$ & -2.14867800 & 1.23325700 & -0.18100800 \\
\hline $\mathrm{C}$ & -0.75987500 & 1.30002000 & -0.47733400 \\
\hline $\mathrm{C}$ & -2.66852100 & -0.07926900 & 0.19215400 \\
\hline $\mathrm{C}$ & -3.99129800 & -0.38431000 & 0.55111700 \\
\hline $\mathrm{C}$ & -4.33946300 & -1.69952500 & 0.83422400 \\
\hline $\mathrm{C}$ & -3.36807100 & -2.70279600 & 0.74721700 \\
\hline $\mathrm{C}$ & -2.07632100 & -2.33661300 & 0.38957900 \\
\hline $\mathrm{N}$ & -1.73818400 & -1.06418900 & 0.13316500 \\
\hline $\mathrm{H}$ & 0.78246400 & 2.60221500 & -1.25326800 \\
\hline $\mathrm{H}$ & -0.65869000 & 4.56971400 & -1.48059900 \\
\hline $\mathrm{H}$ & -3.07346000 & 4.45115300 & -0.85845400 \\
\hline $\mathrm{H}$ & -4.02200600 & 2.31141300 & -0.07095200 \\
\hline $\mathrm{H}$ & -4.73348100 & 0.40309400 & 0.60925400 \\
\hline $\mathrm{H}$ & -5.35947300 & -1.94420000 & 1.11598500 \\
\hline $\mathrm{H}$ & -3.60248700 & -3.74233400 & 0.94723700 \\
\hline $\mathrm{H}$ & -1.28215200 & -3.06931600 & 0.29331300 \\
\hline $\mathrm{Ru}$ & 0.28332500 & -0.40957700 & -0.14687000 \\
\hline $\mathrm{H}$ & 0.89803300 & 0.37323000 & -1.31687300 \\
\hline $\mathrm{Cl}$ & 0.24637800 & -0.08839700 & 2.24660300 \\
\hline $\mathrm{Cl}$ & 0.11970800 & -1.70134800 & -2.17555600 \\
\hline $\mathrm{C}$ & 4.32505400 & 0.87111800 & 0.66411200 \\
\hline $\mathrm{C}$ & 4.56446500 & -0.36021600 & 0.15158700 \\
\hline $\mathrm{H}$ & 4.99572800 & 1.61579100 & 1.06278500 \\
\hline $\mathrm{H}$ & 5.48388000 & -0.90839200 & 0.01994600 \\
\hline $\mathrm{C}$ & 2.34086100 & 2.29385600 & 1.12910900 \\
\hline $\mathrm{H}$ & 1.27133000 & 2.14457000 & 1.23415500 \\
\hline $\mathrm{H}$ & 2.76140500 & 2.50029900 & 2.1160190 \\
\hline $\mathrm{H}$ & 2.54592800 & 3.13461900 & 0.45998600 \\
\hline $\mathrm{C}$ & 3.19568300 & -2.25400500 & -0.71795700 \\
\hline $\mathrm{H}$ & 4.06778100 & -2.49399200 & -1.33024800 \\
\hline $\mathrm{H}$ & 3.14098700 & -2.95402500 & 0.12213100 \\
\hline $\mathrm{H}$ & 2.29830400 & -2.33349500 & -1.32977500 \\
\hline C & 2.32215800 & -0.00907300 & 0.06266800 \\
\hline $\mathrm{N}$ & 2.95468400 & 1.07289000 & 0.60610600 \\
\hline $\mathrm{N}$ & 3.33581500 & -0.88352300 & -0.2217310 \\
\hline
\end{tabular}

- 3t-c

\section{$\mathrm{E}=-1173.6518469 \mathrm{Ha}$}

$\begin{array}{ll}C & 0.38298200 \\ C & -0.07833500 \\ C & -1.41127900 \\ C & -2.28085300 \\ C & -1.83417600 \\ C & -0.46807300 \\ C & -2.71003000 \\ C & -4.10693100 \\ C & -4.83093600\end{array}$
1. 61063500
1.93461500
2.06219100
3.16860600
1.84761100
3.54602300
1.18295300
2.68122200
0.71234500
1.43688200
0.89086400
1.07137700
$-0.04584900$
0.52779200
0.06844600
0.49027100
$-0.70647400$
$-0.41260800$ 


$\begin{array}{lr}\mathrm{C} & -4.14605500 \\ \mathrm{C} & -2.75646800 \\ \mathrm{~N} & -2.05908300 \\ \mathrm{H} & 1.37572100 \\ \mathrm{H} & 0.58990600 \\ \mathrm{H} & -1.77343100 \\ \mathrm{H} & -3.30778800 \\ \mathrm{H} & -4.60777800 \\ \mathrm{H} & -5.91314700 \\ \mathrm{H} & -4.67065000 \\ \mathrm{H} & -2.16281000 \\ \mathrm{Ru} & 0.08976400 \\ \mathrm{H} & -0.12562400 \\ \mathrm{Cl} & 0.20259600 \\ \mathrm{Cl} & 0.06747700 \\ \mathrm{C} & 4.09199500 \\ \mathrm{C} & 4.35019200 \\ \mathrm{H} & 4.74273600 \\ \mathrm{H} & 5.27461800 \\ \mathrm{C} & 2.02484700 \\ \mathrm{H} & 1.52320000 \\ \mathrm{H} & 1.29480400 \\ \mathrm{H} & 2.74865300 \\ \mathrm{C} & 3.03555400 \\ \mathrm{H} & 4.01980000 \\ \mathrm{H} & 2.72909300 \\ \mathrm{H} & 2.30573800 \\ \mathrm{C} & 2.11684900 \\ \mathrm{~N} & 2.73011400 \\ \mathrm{~N} & 3.14619100 \\ \mathrm{C} & 0.46917300 \\ \mathrm{O} & 1.05285700 \\ \mathrm{O} & 0.46265200 \\ \mathrm{O} & -0.29251800 \\ \mathrm{H} & -0.17913700 \\ & \\ & \\ \mathrm{H} & \end{array}$

$\begin{array}{rr}-1.57643900 & -1.26490000 \\ -1.63447500 & -1.18584200 \\ -0.89363900 & -0.30915200 \\ 1.86147800 & 1.58235900 \\ 2.61561000 & 3.82284400 \\ 2.20564100 & 4.50606800 \\ 1.00341000 & 2.98763200 \\ 0.77676300 & 1.14091800 \\ -0.62116200 & -0.46079100 \\ -2.19202100 & -1.98824400 \\ -2.28211800 & -1.82043200 \\ -0.87831500 & -0.15692900 \\ 0.96690100 & -0.03600900 \\ -3.25646000 & -0.47676500 \\ -0.41144400 & -2.47854500 \\ -1.12668000 & 1.24412700 \\ -0.35661400 & 0.15573700 \\ -1.51417000 & 2.01231800 \\ 0.06096600 & -0.21053200 \\ -2.10390900 & 2.27586800 \\ -1.40590000 & 2.95618700 \\ -2.77842400 & 1.82397400 \\ -2.69385400 & 2.84390500 \\ 0.69422800 & -1.69519100 \\ 1.13293400 & -1.87738500 \\ 0.08901800 & -2.54764000 \\ 1.49207800 & -1.51335300 \\ -0.77633700 & 0.14932600 \\ -1.38097500 & 1.22471400 \\ -0.14896200 & -0.49658500 \\ 3.78933600 & -1.30471600 \\ 2.86220400 & -0.65692900 \\ 5.01317500 & -1.11901600 \\ 3.33534900 & -2.41006600 \\ 2.37129500 & -2.43297000\end{array}$

\section{- 4t-c}

\section{$\mathrm{E}=-1173.64524669 \mathrm{Ha}$}

$\begin{array}{lrrr}\text { C } & 0.40579100 & 1.93876700 & 1.66622900 \\ \mathrm{C} & -0.02717700 & 2.52409000 & 2.85239100 \\ \mathrm{C} & -1.29346300 & 2.21778900 & 3.37262700 \\ \mathrm{C} & -2.10926800 & 1.30990200 & 2.69876200 \\ \mathrm{C} & -1.67785600 & 0.70111600 & 1.51067500 \\ \mathrm{C} & -0.38346700 & 0.99239200 & 0.95879500 \\ \mathrm{C} & -2.47180400 & -0.33591400 & 0.83663000 \\ \mathrm{C} & -3.86086300 & -0.49633400 & 0.94319500 \\ \mathrm{C} & -4.48194500 & -1.53827900 & 0.25966900 \\ \mathrm{C} & -3.70545800 & -2.40306600 & -0.51679800 \\ \mathrm{C} & -2.33122600 & -2.18555000 & -0.58754500 \\ \mathrm{~N} & -1.73276500 & -1.17998200 & 0.07045900 \\ \mathrm{H} & 1.35759600 & 2.23891800 & 1.23975500 \\ \mathrm{H} & 0.61009900 & 3.24170900 & 3.36447800 \\ \mathrm{H} & -1.63740300 & 2.68028400 & 4.29398400 \\ \mathrm{H} & -3.07627000 & 1.04641200 & 3.12027700 \\ \mathrm{H} & -4.44202100 & 0.20642700 & 1.53011300 \\ \mathrm{H} & -5.55948900 & -1.66611600 & 0.32086100 \\ \mathrm{H} & -4.15044200 & -3.22477300 & -1.06848500 \\ \mathrm{H} & -1.67048500 & -2.81544500 & -1.17183000 \\ \mathrm{Ru} & 0.36776200 & -0.71038700 & -0.01216400\end{array}$




$\begin{array}{lrrr}\mathrm{H} & -0.26613700 & 1.25646300 & -0.36180500 \\ \mathrm{Cl} & 0.94776800 & -3.08809100 & 0.13437100 \\ \mathrm{Cl} & 0.14023500 & -0.85984800 & -2.37794300 \\ \mathrm{C} & 4.46891000 & 0.13002000 & 0.75096700 \\ \mathrm{C} & 4.37367200 & 0.78300200 & -0.43542100 \\ \mathrm{H} & 5.29857900 & 0.00845400 & 1.42987000 \\ \mathrm{H} & 5.10808800 & 1.33896300 & -0.99689100 \\ \mathrm{C} & 2.87997900 & -1.12335300 & 2.22769000 \\ \mathrm{H} & 2.35651200 & -0.44975500 & 2.91612700 \\ \mathrm{H} & 2.24962900 & -1.98287600 & 1.99256600 \\ \mathrm{H} & 3.80146500 & -1.47299400 & 2.70082400 \\ \mathrm{C} & 2.59961300 & 1.23046300 & -2.13452500 \\ \mathrm{H} & 3.29147000 & 2.03688100 & -2.39370300 \\ \mathrm{H} & 2.57406800 & 0.48661000 & -2.93240200 \\ \mathrm{H} & 1.59834300 & 1.64107700 & -1.99347700 \\ \mathrm{C} & 2.32775100 & -0.13324500 & -0.01423900 \\ \mathrm{~N} & 3.22351300 & -0.42699400 & 0.99306900 \\ \mathrm{~N} & 3.07172000 & 0.62467800 & -0.88521800 \\ \mathrm{C} & -1.35183100 & 2.67257200 & -1.92180400 \\ \mathrm{O} & -0.28720700 & 2.35286600 & -1.24606200 \\ \mathrm{O} & -1.83553900 & 3.79455600 & -2.05253300 \\ \mathrm{O} & -1.98086500 & 1.61943200 & -2.55940200 \\ \mathrm{H} & -1.39771900 & 0.83677800 & -2.50227200\end{array}$

\section{- 5t-c}

\section{$\mathrm{E}=-1173.6982737 \mathrm{Ha}$}

$\begin{array}{lrrr}\mathrm{C} & -0.43132700 & -0.68056800 & 2.31890200 \\ \mathrm{C} & 0.21848400 & -0.75651700 & 3.54946100 \\ \mathrm{C} & 1.37306600 & 0.00313500 & 3.80117900 \\ \mathrm{C} & 1.88090500 & 0.80637500 & 2.78487100 \\ \mathrm{C} & 1.23788700 & 0.88067100 & 1.53511100 \\ \mathrm{C} & 0.02143100 & 0.16569100 & 1.27583700 \\ \mathrm{C} & 1.78227600 & 1.63540300 & 0.40797900 \\ \mathrm{C} & 2.93762400 & 2.43700600 & 0.42786300 \\ \mathrm{C} & 3.35699200 & 3.08174600 & -0.72986000 \\ \mathrm{C} & 2.61216300 & 2.92567000 & -1.90428200 \\ \mathrm{C} & 1.47301700 & 2.12809200 & -1.85710500 \\ \mathrm{~N} & 1.06515100 & 1.49784700 & -0.74449500 \\ \mathrm{H} & -1.30613200 & -1.30182700 & 2.15097500 \\ \mathrm{H} & -0.16637600 & -1.42399200 & 4.31916100 \\ \mathrm{H} & 1.88082400 & -0.05941600 & 4.75988300 \\ \mathrm{H} & 2.79882400 & 1.36282900 & 2.96040300 \\ \mathrm{H} & 3.50153400 & 2.54682100 & 1.34744200 \\ \mathrm{H} & 4.25275400 & 3.69723300 & -0.71917300 \\ \mathrm{H} & 2.90097900 & 3.40734900 & -2.83277200 \\ \mathrm{H} & 0.84824000 & 1.98560300 & -2.73361500 \\ \mathrm{Ru} & -0.75428400 & 0.39053400 & -0.52499500 \\ \mathrm{H} & 1.96650900 & -2.65587000 & 0.06907200 \\ \mathrm{Cl} & -1.89253800 & 2.53280400 & -0.63673100 \\ \mathrm{Cl} & 0.44954400 & -1.69634500 & -1.23960900 \\ \mathrm{C} & -4.63679500 & -0.98446200 & 0.63813300 \\ \mathrm{C} & -4.48582000 & -1.72495900 & -0.48810600 \\ \mathrm{H} & -5.45796700 & -0.91887700 & 1.33549200 \\ \mathrm{H} & -5.14988200 & -2.42713000 & -0.96857600 \\ \mathrm{C} & -3.32219400 & 0.72468400 & 1.87374400 \\ \mathrm{H} & -3.26794400 & 0.21747000 & 2.84285500 \\ \mathrm{H} & -2.41374500 & 1.29542400 & 1.70386900 \\ \mathrm{H} & -4.17593200 & 1.40926500 & 1.86179800 \\ \mathrm{C} & -2.77871200 & -1.92224200 & -2.28752800 \\ & & & \end{array}$




$\begin{array}{lrrr}\mathrm{H} & -3.06862400 & -2.97190000 & -2.39811400 \\ \mathrm{H} & -3.23527600 & -1.33654100 & -3.09507200 \\ \mathrm{H} & -1.69429400 & -1.85158000 & -2.34426900 \\ \mathrm{C} & -2.55525700 & -0.50867900 & -0.20617200 \\ \mathrm{~N} & -3.47271500 & -0.24895000 & 0.79692400 \\ \mathrm{~N} & -3.22575600 & -1.43348000 & -0.98994900 \\ \mathrm{C} & 3.85938100 & -2.64257100 & -0.39264300 \\ \mathrm{O} & 2.81898400 & -3.05417100 & 0.35853400 \\ \mathrm{O} & 4.98836400 & -3.02795400 & -0.18405300 \\ \mathrm{O} & 3.57617900 & -1.78226900 & -1.39119700 \\ \mathrm{H} & 2.61222300 & -1.58178400 & -1.44972400\end{array}$

\section{Structure of $\mathrm{HCO}_{3}^{-}$, d, e and f}

\section{$\mathrm{HCO}_{3}^{-}$}

\section{$\mathrm{E}=-264.4727409 \mathrm{Ha}$}

$\begin{array}{rrrr}\mathrm{C} & 0.156696 & 0.067523 & -0.000030 \\ \mathrm{O} & 1.228288 & -0.559696 & 0.000198 \\ \mathrm{O} & -1.021895 & -0.773386 & -0.000445 \\ \mathrm{H} & -1.740196 & -0.125613 & 0.003260 \\ \mathrm{O} & -0.106390 & 1.298142 & -0.000139\end{array}$

\section{- 1t-d}

\section{$\mathrm{E}=-909.1410267 \mathrm{Ha}$}

$\begin{array}{lrrr}\mathrm{C} & 2.56494300 & -1.32678600 & 0.56204400 \\ \mathrm{C} & 3.81147800 & -1.01067400 & 1.08801300 \\ \mathrm{C} & 4.15452300 & 0.33595600 & 1.24600300 \\ \mathrm{C} & 3.25112000 & 1.31026000 & 0.83414200 \\ \mathrm{C} & 2.01154800 & 0.93116700 & 0.29835500 \\ \mathrm{C} & 1.03793800 & 1.85384500 & -0.29197600 \\ \mathrm{C} & 1.09448300 & 3.24848400 & -0.16122200 \\ \mathrm{C} & 0.17858000 & 4.05906600 & -0.83268900 \\ \mathrm{C} & -0.79660300 & 3.48360100 & -1.65810500 \\ \mathrm{C} & -0.88183500 & 2.09720200 & -1.77902900 \\ \mathrm{H} & 2.26138700 & -2.35313700 & 0.39102400 \\ \mathrm{H} & 4.49859600 & -1.80704500 & 1.35254300 \\ \mathrm{H} & 5.11955300 & 0.61939700 & 1.65467000 \\ \mathrm{H} & 3.51280000 & 2.36047100 & 0.89339700 \\ \mathrm{H} & 1.85158800 & 3.70953200 & 0.46596300 \\ \mathrm{H} & 0.23640300 & 5.13838500 & -0.72838100 \\ \mathrm{H} & -1.48495300 & 4.11739800 & -2.21000900 \\ \mathrm{H} & -1.61882900 & 1.64575000 & -2.43500800 \\ \mathrm{Ru} & -0.20840600 & -0.86736900 & -0.58571400 \\ \mathrm{Cl} & 1.03422700 & -2.07904400 & -2.29106300 \\ \mathrm{Cl} & -2.23731200 & -1.88180100 & -1.34164400 \\ \mathrm{C} & -1.29827100 & -0.24455600 & 1.07786300 \\ \mathrm{~N} & -2.46322000 & 0.46503100 & 1.19094500 \\ \mathrm{C} & -2.87819200 & 0.55805500 & 2.50980600 \\ \mathrm{C} & -1.96975400 & -0.11389000 & 3.25834900 \\ \mathrm{~N} & -1.01534800 & -0.59835100 & 2.37590800 \\ \mathrm{C} & -3.26622300 & 1.01201700 & 0.09752100 \\ \mathrm{C} & 0.10215200 & -1.42811100 & 2.81489200 \\ \mathrm{H} & -3.78377800 & 1.07216100 & 2.79087800 \\ \mathrm{H} & -1.92513500 & -0.30337700 & 4.31919800 \\ \mathrm{H} & -4.32175300 & 0.87310700 & 0.34316000 \\ \mathrm{H} & -3.05814500 & 2.07636000 & -0.03508100 \\ & & & \end{array}$




$\begin{array}{rrrr}\mathrm{H} & 0.29706300 & -2.20614900 & 2.07694800 \\ \mathrm{H} & 1.00569000 & -0.83147800 & 2.95964500 \\ \mathrm{H} & -0.16887000 & -1.90559300 & 3.75881800 \\ \mathrm{H} & -3.04676600 & 0.45302500 & -0.80894400 \\ \mathrm{C} & 0.00112200 & 1.26536300 & -1.06679300 \\ \mathrm{H} & 0.26472800 & 0.28936900 & -1.71813800 \\ \mathrm{~N} & 1.66152200 & -0.38605000 & 0.21510500\end{array}$

\section{- 1t-e}

\section{$\mathrm{E}=-909.1526981 \mathrm{Ha}$}

\begin{tabular}{|c|c|c|c|}
\hline C & 0.02274400 & 2.50839400 & -1.00234900 \\
\hline $\mathrm{C}$ & -0.65787000 & 3.72396700 & -1.00543600 \\
\hline C & -2.01248000 & 3.76845600 & -0.65520300 \\
\hline $\mathrm{C}$ & -2.68528900 & 2.59095100 & -0.32912200 \\
\hline $\mathrm{C}$ & -2.02373500 & 1.35463100 & -0.33403200 \\
\hline C & -0.62897900 & 1.31579200 & -0.62882800 \\
\hline $\mathrm{C}$ & -2.69287400 & 0.08747700 & -0.00724400 \\
\hline $\mathrm{C}$ & -4.08221500 & -0.07737600 & 0.09974500 \\
\hline C & -4.60491400 & -1.33023100 & 0.40293700 \\
\hline $\mathrm{C}$ & -3.73182800 & -2.40541700 & 0.58589400 \\
\hline $\mathrm{C}$ & -2.36558600 & -2.17734000 & 0.45758900 \\
\hline $\mathrm{N}$ & -1.85816900 & -0.96761800 & 0.17583300 \\
\hline $\mathrm{H}$ & 1.06336300 & 2.46515000 & -1.30560300 \\
\hline $\mathrm{H}$ & -0.13700000 & 4.63437500 & -1.28850500 \\
\hline $\mathrm{H}$ & -2.54461200 & 4.71480000 & -0.64503400 \\
\hline $\mathrm{H}$ & -3.73381400 & 2.64332700 & -0.05344000 \\
\hline $\mathrm{H}$ & -4.74459600 & 0.76427200 & -0.06513200 \\
\hline $\mathrm{H}$ & -5.67863500 & -1.46811700 & 0.48819400 \\
\hline $\mathrm{H}$ & -4.09509900 & -3.40067100 & 0.81721600 \\
\hline $\mathrm{H}$ & -1.64297800 & -2.97688800 & 0.57995200 \\
\hline $\mathrm{Ru}$ & 0.23863800 & -0.55783000 & 0.04467300 \\
\hline $\mathrm{H}$ & -0.18295100 & 0.41277700 & -1.28256300 \\
\hline $\mathrm{Cl}$ & 0.27386500 & -0.42168100 & 2.42415900 \\
\hline $\mathrm{Cl}$ & 0.16904800 & -1.79979800 & -2.05396000 \\
\hline $\mathrm{C}$ & 4.25246100 & 0.95714300 & 0.47628300 \\
\hline C & 4.54314100 & -0.21274100 & -0.14115200 \\
\hline $\mathrm{H}$ & 4.89345500 & 1.74235300 & 0.84568600 \\
\hline $\mathrm{H}$ & 5.48835100 & -0.65366400 & -0.41661900 \\
\hline $\mathrm{C}$ & 2.22991900 & 2.10513300 & 1.32520900 \\
\hline $\mathrm{H}$ & 1.16405800 & 1.91800100 & 1.39120600 \\
\hline $\mathrm{H}$ & 2.63726500 & 2.13884600 & 2.33901200 \\
\hline $\mathrm{H}$ & 2.41589800 & 3.06023400 & 0.82458000 \\
\hline C & 3.29418200 & -2.20693600 & -0.92181200 \\
\hline $\mathrm{H}$ & 4.03153300 & -2.30384300 & -1.72296800 \\
\hline $\mathrm{H}$ & 3.53518600 & -2.91511400 & -0.12188100 \\
\hline $\mathrm{H}$ & 2.30657600 & -2.41576300 & -1.32531300 \\
\hline $\mathrm{C}$ & 2.26952100 & -0.09004300 & 0.03717100 \\
\hline $\mathrm{N}$ & 2.87175400 & 1.01845100 & 0.58706000 \\
\hline $\mathrm{N}$ & 3.33459300 & -0.83962700 & -0.4022790 \\
\hline
\end{tabular}

\section{- 1t-f}

$\mathrm{E}=-909.15764061 \mathrm{Ha}$

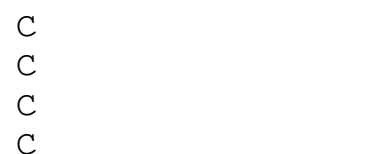

1.48361700

1.98575200

1. 36792700

0.24621500
2.17778500

3.37588700

3.97097000

3.37990700
$-1.78673500$

$-1.27465000$

$-0.16875000$

0.41546600 


\begin{tabular}{|c|c|c|c|}
\hline C & -0.27390200 & 2.18097300 & -0.09606700 \\
\hline $\mathrm{C}$ & 0.37254900 & 1.57112400 & -1.19218400 \\
\hline $\mathrm{C}$ & -1.45478200 & 1.51519800 & 0.47729700 \\
\hline $\mathrm{C}$ & -2.48441500 & 2.20253300 & 1.13150800 \\
\hline $\mathrm{C}$ & -3.60978300 & 1.51724300 & 1.57911600 \\
\hline $\mathrm{C}$ & -3.69290000 & 0.14268400 & 1.33871700 \\
\hline $\mathrm{C}$ & -2.64789900 & -0.49921100 & 0.68889700 \\
\hline $\mathrm{N}$ & -1.53058200 & 0.16105000 & 0.29244800 \\
\hline $\mathrm{H}$ & 1.93759400 & 1.72188700 & -2.66138800 \\
\hline $\mathrm{H}$ & 2.84761100 & 3.84800100 & -1.73712400 \\
\hline $\mathrm{H}$ & 1.76113500 & 4.89692100 & 0.24062100 \\
\hline $\mathrm{H}$ & -0.20781100 & 3.84277600 & 1.28662400 \\
\hline $\mathrm{H}$ & -0.14939400 & 0.78308600 & -1.79777000 \\
\hline $\mathrm{H}$ & -2.40909700 & 3.27771200 & 1.24942800 \\
\hline $\mathrm{H}$ & -4.41581000 & 2.04681100 & 2.07705200 \\
\hline $\mathrm{H}$ & -4.56127300 & -0.43454200 & 1.63731000 \\
\hline $\mathrm{H}$ & -2.65845900 & -1.55975600 & 0.47135800 \\
\hline $\mathrm{Ru}$ & -0.11907500 & -0.81343100 & -0.72858300 \\
\hline $\mathrm{Cl}$ & -1.47598500 & -1.13893300 & -2.73058900 \\
\hline $\mathrm{Cl}$ & -0.46296100 & -3.03567700 & 0.01554300 \\
\hline $\mathrm{C}$ & 1.34692100 & -0.72096800 & 0.68917100 \\
\hline $\mathrm{N}$ & 1.30040600 & -0.75000300 & 2.05736700 \\
\hline $\mathrm{C}$ & 2.57425500 & -0.80332100 & 2.60731200 \\
\hline $\mathrm{C}$ & 3.45093500 & -0.81200900 & 1.57249100 \\
\hline $\mathrm{N}$ & 2.69116800 & -0.75659500 & 0.41238500 \\
\hline $\mathrm{C}$ & 0.07958400 & -0.83261600 & 2.85525600 \\
\hline $\mathrm{C}$ & 3.26001700 & -0.89722700 & -0.92225300 \\
\hline $\mathrm{H}$ & 2.74010400 & -0.83119100 & 3.67277400 \\
\hline $\mathrm{H}$ & 4.52873200 & -0.85432300 & 1.55755200 \\
\hline $\mathrm{H}$ & 0.35432000 & -1.10516400 & 3.87596000 \\
\hline $\mathrm{H}$ & -0.44348100 & 0.12610600 & 2.87298100 \\
\hline $\mathrm{H}$ & 2.49661000 & -0.64477400 & -1.65943900 \\
\hline $\mathrm{H}$ & 4.10740900 & -0.21657500 & -1.04153800 \\
\hline $\mathrm{H}$ & 3.58905300 & -1.92707000 & -1.09206300 \\
\hline $\mathrm{H}$ & -0.56736600 & -1.60547800 & 2.43811700 \\
\hline
\end{tabular}



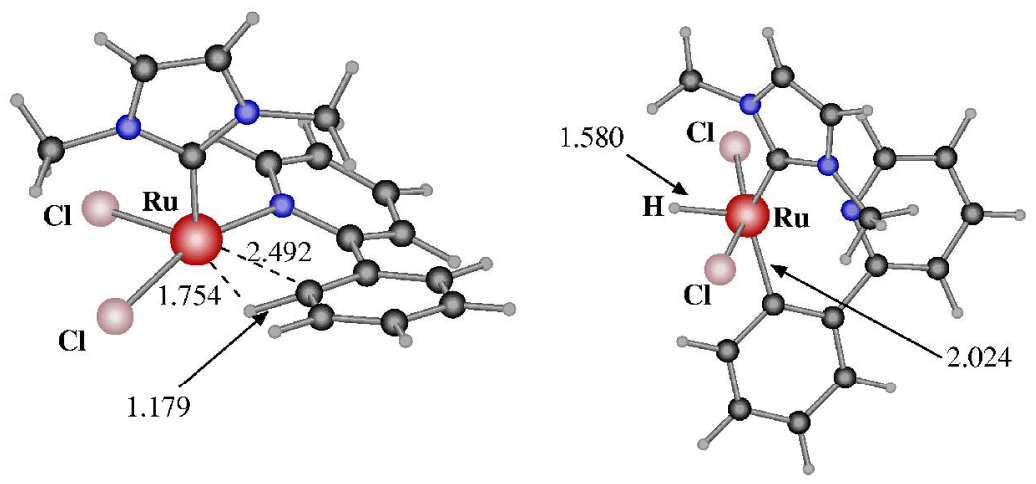

$$
\text { 1t-a 2t-a }
$$

Figure S1. B3LYP-optimized structures for complexes 1t-a, 2t-a. Selected distances are indicated in $\AA$. 